\title{
A CLINICAL CONTRIBUTION TO THE STUDY OF THE AETIOLOGY OF THE FIBROSITIC NODULE
}

\author{
BY W. S. C. COPEMAN
}

THe term " nodule" is employed in the title of this paper, as it is one that has become familiarised by custom. It has seemed, however, that the existence of a nodule sufficiently definite to be palpated in the muscles of a sufferer with fibrositis must in most cases be a sign of chronicity of the complaint, and that the "myalgic spot" is probably an earlier stage of what later becomes a nodule if left untreated. The discrepancy in the frequency with which nodules are found in this disease in England and in America can be explained by this hypothesis.

The myalgic spot is an area of hyperæsthesia in the substance of a muscle or its tendinous sheath which gives rise to pain either in the same locality or referred to a distance when stimulated. We owe mostly to Kellgren the description of this referred pain and its distribution. These spots are generally multiple and occur in the powerful muscles of the back and of the gluteal region; often towards the edge of the muscle, where it blends with the more fibrinous portion. They may also occur, but less frequently, in the peripheral muscles, and in the anterior muscles of the trunk.

The ætiology and pathology of the myalgic spot or nodule is unknown, although trauma is believed by most observers to play a part in its development. It is thought that the observations recorded in this paper throw some light on the ætiology in a proportion of cases.

\section{Observations on Acute Myalgia}

An epidemic of influenza was recently studied in which severe pain in the lower back was an early and prominent symptom in nearly every case. Some patients also complained later of pains in the thighs, calves, and arms.

The cases comprising this epidemic were all of the "febrile" type described by Horder and Gow (Price's " Medicine," 1941) as being of abrupt onset, with pyrexia and associated malaise and headache in addition to the pains described above. The pyrexia was high for three to five days and accompanied by a moderate degree of catarrh chiefly affecting the eyes. Defervescence was by rapid lysis, and a leucopenia was present in most cases. None of the forty patients examined had any previous history of rheumatism.

It was noted in the course of this epidemic that although the acute lumbar pain was diffuse, severe, and resulted in spasm in some cases, by careful examination it could be found that it radiated from localised 
hypersensitive areas in the adjacent muscles; and that the position of these areas approximated to those usual in cases of ordinary lumbago. The same observation was made in cases where the pain was in the limbs.

In one instance also severe " crampy" pain in the upper portion of the rectus abdominis muscle was met with-the clinical picture being comparable with Bornhölm disease. This pain was entirely relieved by an injection of procain at the focal point.

The subjective pain disappeared after two or three days, but these myalgic spots were found by palpation to be still present, although the patient was not aware of their presence. When they were found and pressed on he experienced a sudden sharp pain which made him wince momentarily and which was-in about half the cases examinedreferred in addition to the whole area previously affected.

The number of such spots which could be found in any patient tended progressively to decrease as time went in.

The same group of patients were again examined two months after their influenza, when it was found that only in rather less than half of them could these spots still be found. In these cases, as before, the patient was himself unaware of their presence until examination.

Three cases deserve special mention.

Case 1:A Civilian aged 40.--Sudden onset of headache with pain behind the eyes, sweating and malaise. Pain in the back had occurred several hours previously, and this got progressively worse. The patient preferred walking about rather than lying in bed for this reason, although his temperature was $102 \cdot 6$.

On examination the patient looked ill and was sweating profusely. The eyes were congested and the tongue furred. Pain in the back was evidently severe and the muscles appeared to be in spaem.

Urine and stools, normal. Blood; no malarial parasites W.B.C. 5,000.

Next day the patient was much better. There was still pain behind the eyes and in the back. The temperature was normal. Myalgic spots were found in the lumbar region. The patient made an uninterrupted recovery, but three weeks later he developed mumps. The pain then returned to the lumbar region as at first, although not so severely, for two days during which the temperature was raised above $100^{\circ} \mathrm{F}$.

Case 2 : A Civilian aged 27.-Complaining of sudden onset of severe pains in head, back, and legs, with feeling of pyrexia, previous afternoon. Temperature was 101.6, pulse 90. When seen, he was sweating profusely. No shivering; bowels rather loose on previous day but now constipated. Patient looked ill tongue lightly furred. On examination: abdomen-nothing abnormal found; blood-no malarial parasites, W.B.C. 3,400. The pains had prevented much sleep previously, but sedatives gave some relief.

One week later pain and other symptoms had entirely gone, and patient had resumed work. Pain could, however, be provoked momentarily by pressure on the myalgic spots which still persisted in lumbar region and along the border of the ilium on both sides. Two weeks later the condition was unchanged, excepting that fewer of these spots could be found. Those present were carefully marked.

Five c.c. of blood, taken in the first 12 hours, from a patient who had just developed sandfly fever, was then injected intramuscularly-by permission- 
and on the next day a pyrexial attack with a maximum temperature of $101^{\circ}$ developed and lasted 36 hours. The patient complained of lumbago from the onset of this pyrexia, and on examination it was found that this pain was originating from the spots which had been marked. It was easily abolished by injecting 1 per cent. novocaine into them.

One month later this patient was still found to have three myalgic spots in the lumbar region of which he was completely unaware.

Case 3 : A Gunner aged 22.- Sudden onset of severe occipital headache with pains in back and legs. Temperature $101^{\circ}$.

On examination patient looked and felt very ill. Marked congestion of the conjunctivæ; the bowels had been rather loose for two days. Tongue moist with light brown fur. Abdomen-normal. Stools and urine-normal. W.B.C. 4,200 .

Pains disappeared on third day; temperature normal and all symptoms gone on fifth day.

Two weeks later patient stated when seen that he was perfectly well, but well-defined myalgic spots were found in lumbar region. On pressure these referred a pain down the legs entirely comparable with that which had occurred during onset of fever.

This patient was seen again a month later for another reason, and volunteered the information that similar pains had returned, although in a lesser degree, for a short period a few days previously whilst he was starting a cold in the head.

From these observations it seems that infection with the organisms of influenza will produce acute muscular pain which can be shown to be referred from myalgic spots which develop in the region of the affected muscles. The spots appear to be comparable with those found in cases of ordinary lumbago. A large proportion of the spots which were seen to develop in the present series of cases disappeared a few days after the termination of the influenza. A proportion, however, survived.

It does not seem that these myalgic spots are a specific reaction to the virus of influenza as such, however, in view of the fact that the pain returned temporarily in the same areas during an attack of mumps which one of the patients (Case 1) developed spontaneously during convalescence.

This view was confirmed experimentally by inducing an attack of sandfly fever artificially in a patient convalescent from influenza (Case 2), with the same result.

It also seems possible (Case 3) that periodical attacks of the common cold or other mild virus infection may prove to be a factor in keeping active myalgic spots which have arisen in this way, although confirmatory evidence is lacking. If this were found to be so it suggests an analogy with the " maintenance dose" in other fields.

\section{Observations on Myalgia following Exanthemata}

It is well known that acute muscular pains may arise in the course of, or after, many of the acute infectious diseases other than influenza; chiefly it would seem those of virus origin. A considerable number of patients complaining of pain that had arisen in this way have been examined during the past year. These pains have also in the majority of cases been found to have their origin in well defined hypersensitive myalgic spots from which the patient's myalgia or "neuritis" (muscular tenderness) was referred. The diseases which most commonly give rise to this syndrome appear to be sandfly, glandular, undulant and scarlet fevers, measles and rubella.

Myalgic spots arising during the course of one of these diseases have 
been found to be still present two months later; this being the longest period during which "follow up" proved possible in this series.

The description already given of the myalgic spots arising during the epidemic of influenza applies equally to those arising in this series and justifies the belief that they may arise during the course of almost any acute infectious fever, and that a certain proportion will persist unknown to the patient until such time as they may subsequently become reactivated.

It seems probable that such factors as chill, damp, trauma and mild focal infection, which have sometimes been held to be causative, are in fact of secondary importance, and will only provoke an attack of fibrositis or myalgia in the presence of latent myalgic spots formed previously in this way. The method whereby they actually provoke an attack may possibly be by inducing a local œdema in the sensitive area whereby the victim becomes aware of the myalgic spot and of the pain referred from it. If this is so it accounts for the nodular feeling which is not infrequently remarked at these points, and the fact that this can sometimes be rubbed away by a skilful masseur.

\section{SUMMARY}

1. The rôle of " myalgic spots" or "rheumatic nodules" in fibrositis is discussed.

2. In a recent epidemic of influenza in which lumbar and other pain was a prominent feature, it was observed that this pain was also referred from small hypersensitive areas in the muscles. These were indistinguishable from " rheumatic" myalgic spots.

On re-examination of the patients after one and two months these spots were still found to be present, although in diminishing numbers. The patient was, however, invariably unaware of this fact until pressure from the examining finger disclosed them. None of these patients had any previous rheumatic history.

3. Myalgic spots which had arisen during influenza were reactivated in one patient by an attack of mumps which arose spontaneously during convalescence. In another case this occurred as the result of the artificial induction of an attack of sandfly fever by the inoculation of infected blood.

It appears therefore that myalgic spots can arise de novo in the course of acute influenza, and that these will in a proportion of cases persist. They can, however, be reactivated by infections of other types. There was evidence in one case suggesting that the common cold provided the means of maintaining the activity of the myalgic spots.

4. Rheumatic pains are a characteristic sequel of many of the exanthemata, mostly those of virus origin. Observation has shown 
that these pains, which tend to arise in a less acute manner, are also generally of the focal type.

5. The final suggestion is therefore that the myalgic spots which it is now widely agreed form the basis of many of the rheumatic syndromes of later life are sometimes a legacy from acute infections in earlier life. It is important to remember that the victims are often unaware of their presence until they become activated by factors such as chill, trauma, or focal sepsis. These factors should therefore be regarded as secondary and no longer causative in such cases.

My thanks are due to the D.M.S. PAIFORCE and to Maj.-General R. Barnsley, M.C., D.D.M.S. Southern Command, for permission to publish this paper, and to Dr. H. Jamieson, C.M.O. of the A.I.O.C., for help.

\section{NEW PATHS OF INVESTIGATION FOR DIAGNOSIS AND THERAPY}

\section{BY DR. ERNST FREUND}

Strck 1828, when Woehler discovered a method of producing urea in the test tube, thereby proving that some of the substances of organic life could be produced in vitro, pathological investigation has progressed towards a more precise understanding of the activity of the human organism in the sphere of biochemistry.

The study of chemical phenomena, aiming at qualitative and quantitative determinations of the waste products of metabolism, was thus encouraged with a view to the possibility of distinguishing between pathological and normal metabolism, and of this becoming the basis of diagnosis and therapy. There can be no doubt that such a development offers considerably better prospects of effective therapy than the empirical employment of substances of the mineral, plant and animal worlds, dating from the earliest age of medicine (admittedly with very beneficial results in some aspects of the art of healing).

Progress in development must necessarily be sectional. A complete survey of the vast range of biochemical problems involved in the utilisation of varied substances by the human organism, and the waste products of the process of utilisation, is a task of such magnitude that it can be only usefully approached little by little. This paper will deal with certain aspects of diagnosis and therapy arising from a comparison of the effects on the cells of material in the urine coming respectively from $(a)$ healthy and $(b)$ morbid subjects.

We can easily ascertain how much of the ingested food is employed 